\title{
An experimental study of the dimple/gimbal interface in a hard disk drive
}

\author{
Longqiu Li $\cdot$ Edmund Fanslau $\cdot$ Frank Talke
}

Received: 29 August 2010/Accepted: 29 December 2010/Published online: 13 January 2011

(C) The Author(s) 2011. This article is published with open access at Springerlink.com

\begin{abstract}
The contact of the dimple/gimbal interface in a hard disk drive was studied experimentally. Two types of dimples with different surface roughness and several types of gimbal materials were investigated. The load-displacement curves for the contact of the dimple and the gimbal exhibit hysteresis, which is related to the plastic deformation of the asperities, during the first few load/unload cycles. The roughness of dimple and gimbal samples was measured before and after load-unload testing using an AFM. The plasticity index was determined based on the roughness measurement. The results show that the roughness and plasticity index of the non-polished dimple decrease with the number of load/unload cycles significantly, i.e., the contact surface becomes smoother than the original surface due to plastic deformation. The roughness and plasticity index of the laser-polished dimple change slightly before and after the load-unload test.
\end{abstract}

\section{Introduction}

During operation of a hard disk drive (HDD), a suspension spring with a spherical protrusion (dimple) is loaded

\section{Li $(\bowtie)$}

Harbin Institute of Technology, No. 92 West Dazhi St, Harbin, Heilongjiang 150001, China

e-mail: longqiuli@g.mail.com

L. Li · F. Talke

Center for Magnetic Recording Research, University of California, San Diego, 9500 Gilman Dr, La Jolla, CA 92093, USA

E. Fanslau

NHK International Corp, 2350 Mission College Blvd, Suite 1090, Santa Clara, CA 95054, USA against a flat gimbal spring to which the magnetic head is attached. In a HDD, this arrangement permits roll and pitch motion of the slider in addition to a vertical degree of freedom (Fig. 1). Raeymaekers et al. (2010) observed that fretting wear occurs at the interface between the dimple and the gimbal as a function of the relative displacement between dimple and gimbal. Fretting wear of the dimple is likely to generate wear particles and cause failure of the HDD. Wear can also occur at the dimple/gimbal interface during load-unload of the slider on the disk. Lee et al. (2004) investigated wear of HDD and observed that wear particles can lead to failure of the head disk interface. Hence, investigation of the contact between the dimple and the gimbal is important in understanding the generation of wear particles in the dimple/gimbal interface of a HDD.

Several studies have been performed in the past to investigate the mechanics of the dimple/gimbal interface. Li et al. (2009) numerically studied the dimple/gimbal interface by modeling the contact as a spherical shell contacting a rigid flat. They investigated the effect of dimple/gimbal geometry and material properties on the onset of plastic deformation at the dimple/gimbal interface. Lee et al. (2009) investigated slip between a dimple and a flexure for the special case of ramp loading. They developed a finite element (FE) model to investigate the slip motion between dimple and gimbal during ramp contact. In their model, the base of the suspension arm was fixed and an impact force was applied to the suspension lift-tab to simulate contact with the ramp.

Zheng et al. (2010) investigated the normal load between the dimple/gimbal interface during a non-operational shock as a function of dimple design parameters and preload. Raeymaekers et al. (2010) investigated fretting wear of the dimple/gimbal interface and found that fretting wear is highly dependent on the normal load. They 


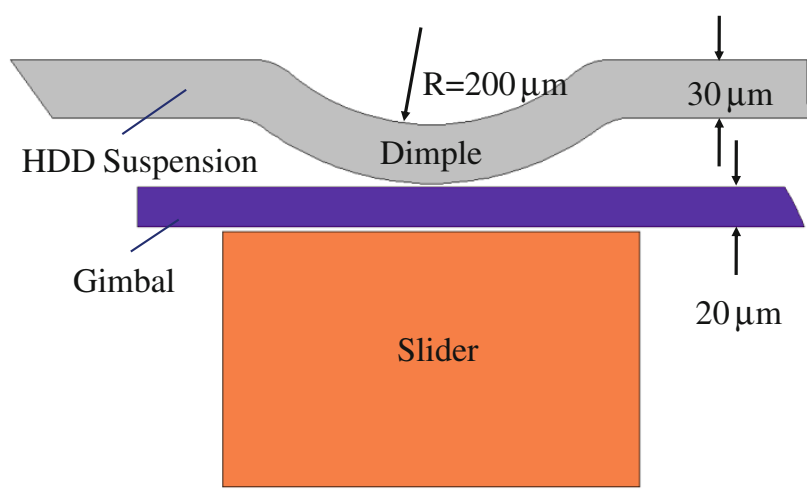

Fig. 1 Schematic representation of the dimple/gimbal contact in a hard disk drive

calculated energy dissipation and plasticity index and found that these parameters were a good measure for resistance to fretting wear. Li et al. (2010a) studied the load process of two different types of dimples against a rigid sapphire gimbal in a nano-indenter. They found that the asperities of the dimple surface deform plastically and are "flattened" after a number of load/unload cycles.

Following the previous work of $\mathrm{Li}$ et al. (2010a), we investigate in this paper the load/unload process and deformation of two types of dimples with different surface roughness for several types of gimbal material using a modified nano-indenter.

\section{Experimental set-up}

Figure 2 presents the basic features of the experimental setup. The test rig is based on a commercially available nano-indenter (Hysitron Inc., USA) in which the tip of the

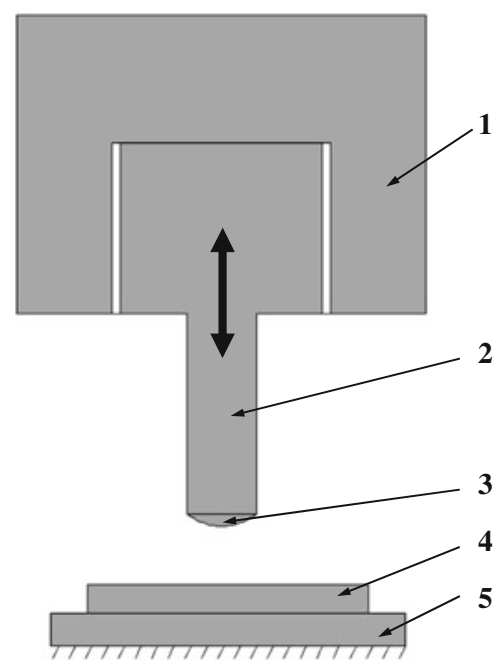

Fig. 2 Schematic representation of the experimental setup: transducer 1 , holder 2 , dimple sample 3 , gimbal sample 4 and stage 5 instrument is replaced by a plastic holder carrying the dimple. The spherical dimple, which was cut from a head suspension, was made of stainless steel (SST304). The dimple sample was glued on the bottom surface of the nano-indenter holder. Three different types of gimbal samples were used. The first gimbal sample was made of sapphire, the second one was made of stainless steel (SST304), and the third one was made of non-polished stainless steel coated with a gold film. The gimbal sample was pressed repeatedly against the dimple sample. The material properties of the dimple and gimbal samples used in the present study are summarized in Table 1 .

The controlled normal load (limited to no more than $9 \mathrm{mN}$ due to the loading range of the nano-indenter) and the corresponding displacement of the dimple were measured using the transducer of the nano-indenter. All experiments were carried out at room temperature of $20-25^{\circ} \mathrm{C}$ and relative humidity of $40-60 \%$. Each experiment was performed on a new dimple and a new contact zone of the gimbal. All experiments were performed under dry condition. Each load/unload cycle was completed in $10 \mathrm{~s}$ ( $5 \mathrm{~s}$ for loading and $5 \mathrm{~s}$ for unloading). There is one thing needs to be noticed that the load/unload process, which was used in present work, is not the same as the park loading/unload process in HDDs. We want to investigate the contact and deformation of dimple/gimbal interface by using the load/unload process in a modified nano-indenter.

\section{Results and discussion}

Figure 3 shows the load-displacement characteristics as a function of load cycles for a non-polished dimple contacting a sapphire gimbal. The curves show 1,2, 5 and 50 cycles. In the first cycle, a large amount of energy dissipation is observed, exhibited by the large area enclosed by the load-displacement curve for a complete load/unload cycle. The reason for this result is that plastic deformation occurs on the asperities of the dimple. We also note from a comparison of the four individual curves of Fig. 3, that plastic deformation decreases with an increase in the number of load/unload cycles.

Table 1 Mechanical properties and roughness of dimples and gimbals

\begin{tabular}{lrlll}
\hline Specimen & $\mathrm{Ra}(\mathrm{nm})$ & $v$ & $Y(\mathrm{GPa})$ & $E(\mathrm{GPa})$ \\
\hline Non-polished dimple & $85 \pm 5$ & 0.31 & 1.68 & 181 \\
Laser polished dimple & $25 \pm 2$ & 0.31 & 1.42 & 181 \\
Sapphire gimbal & $6 \pm 1$ & 0.27 & 2.95 & 435 \\
Non-polished gimbal & $85 \pm 5$ & 0.31 & 1.68 & 190 \\
Gold coated gimbal & $108 \pm 4$ & 0.42 & 0.2 & 77 \\
\hline
\end{tabular}




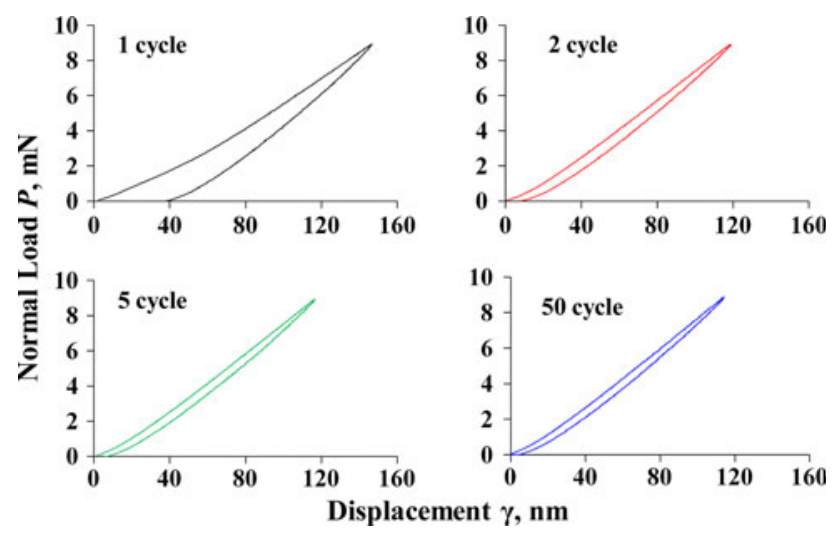

Fig. 3 Normal load versus displacement as a function of load cycles for non-polished dimple

Figure 4 shows the load-displacement characteristics as a function of load cycles for a non-polished dimple (Fig. 4a) and a laser polished dimple (Fig. 4b). We note that the slope of the load-displacement curves becomes steeper with an increase in the number of load/unload cycles, i.e., the dimple/gimbal interface becomes increasingly stiffer. In addition, the dissipated energy decreases (the area enclosed by the load-displacement loop of each cycle) with an increase in the number of cycles. We also observe that the hysteresis is much smaller for the laser
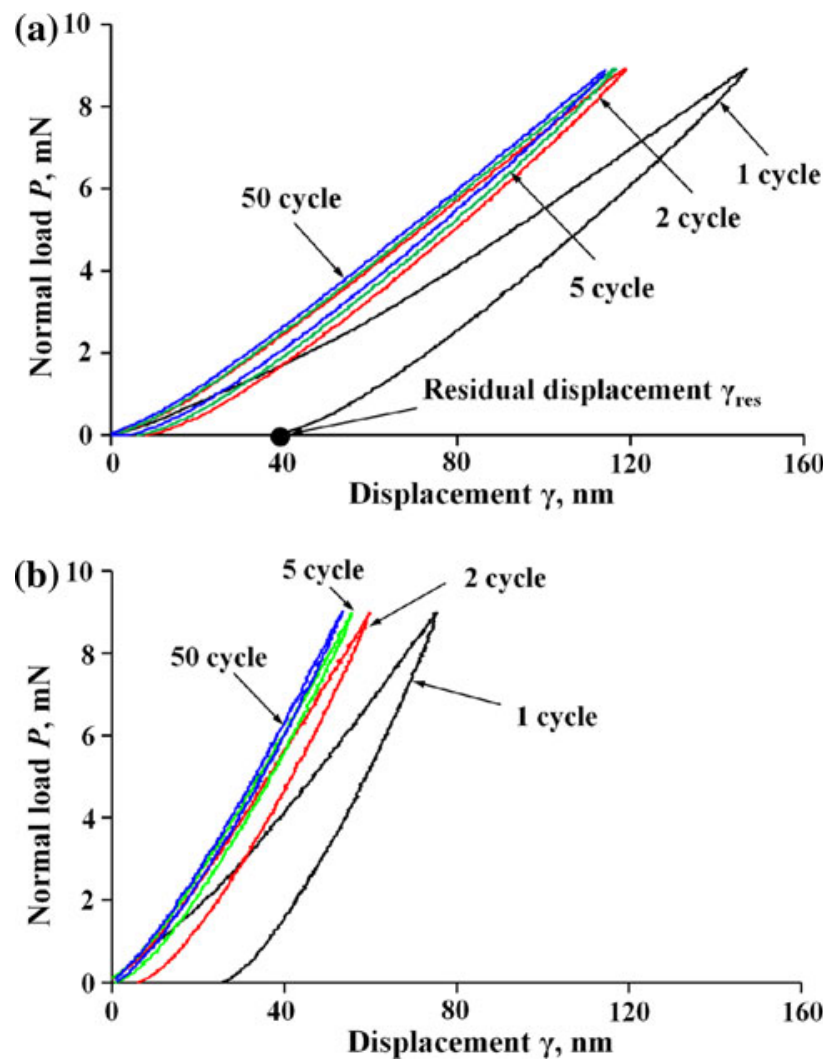

Fig. 4 Normal load versus displacement as a function of load cycles: a non-polished dimple and $\mathbf{b}$ laser polished dimple polished dimple than for the non-polished dimple. Also, the curves do not change very much as a function of the load cycles after 10 cycles. The subsequent cycles exhibit a tendency to "elastic shakedown" (König 1987).

Figure 5 shows that the residual displacement as a function of load/unload cycles for a rough non-polished dimple and a laser polished dimple, respectively. We observe that the residual displacement of both the nonpolished and the laser polished dimple decreases with an increase in the number of load cycles reaching a constant value after 10 cycles.

Figure 6 shows the surface roughness $R_{\mathrm{a}}$ as a function of load/unload cycles for a non-polished dimple and a laser polished dimple, respectively. The roughness of the dimple and gimbal samples was measured using an Atomic Force Microscope (AFM) before and after load-unload testing. The $R_{\mathrm{a}}$ is an arithmetic average value of the peaks and valleys of the scanned surface. We observe that the roughness $R_{\mathrm{a}}$ decreases with an increase in the number of load/unload cycles for the non-polished dimple. However, the surface roughness of a laser polished dimple was found to almost remain constant as functions of the number of load/unload cycles. This is due to the fact that the scan size of the roughness measurement is much larger than the contact area of a laser polished dimple during loading/unloading. The plastically deformed asperities as a result of the load/unload in the laser polished dimple have little contribution to the roughness changes of the whole scanned area.

To characterize rough elastic-plastic contacts, it is common to use the plasticity index ( $\mathrm{Li}$ et al. 2010b).

$\psi=\frac{2 E}{C_{v} \pi\left(1-v^{2}\right) Y}\left(\frac{\sigma_{s}}{\rho}\right)^{0.5}$

where $\sigma_{\mathrm{s}}$ is the standard deviation of asperity summit heights and $\rho$ is the mean asperity radius (equivalent value

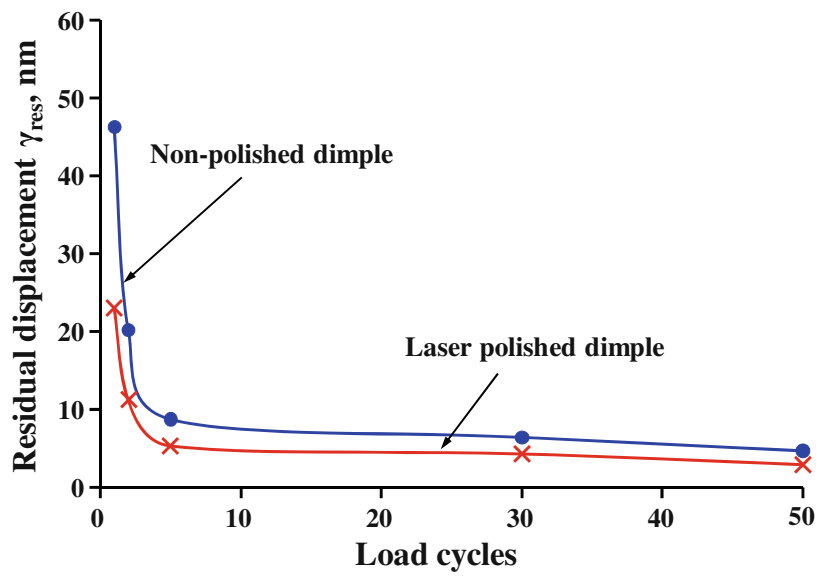

Fig. 5 Residual displacement $\gamma_{\text {res }}$ versus the number of load/unload cycles for non-polished and laser polished dimples after complete unloading from a normal load $P=9 \mathrm{mN}$ 


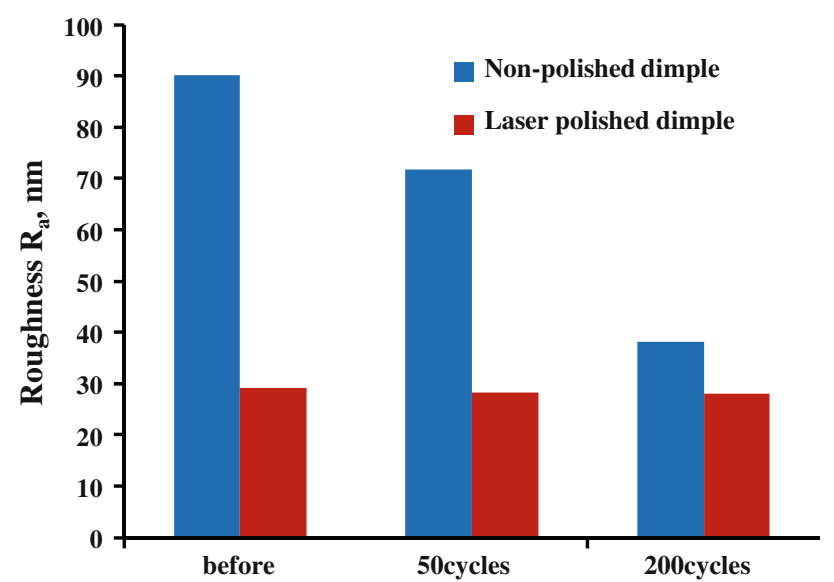

Fig. 6 Roughness $R_{\text {a }}$ value as a function of load/unload cycles for a non-polished dimple and a laser polished dimple

for the combination of two rough surfaces). In Eq. 1, $Y$ is the yield strength and $v$ is the Poisson's ratio of the softer material; $C_{v}=1.234+1.256 v$. The equivalent Young's modulus $E$ is given by.

$\frac{1}{E}=\frac{1-v_{1}^{2}}{E_{1}}+\frac{1-v_{2}^{2}}{E_{2}}$

where $E_{1}, E_{2}, v_{1}$ and $v_{2}$ are the Young's moduli and Poisson's coefficients for both dimple and gimbal material in contact, respectively.

Kogut and Etsion (2003) assumed that a contact is elastic if $\psi<1.4$; that a contact is elastic-plastic if $1.4<\psi<8$; and that a contact is plastic if $\psi>8$. A small plasticity index implies that a surface is smooth and that the asperities are more difficult to deform plastically than in the case that the plasticity index is high. The plasticity index was indentified based on the roughness measurements and material properties shown in Table 1.

Figure 7 shows the plasticity index as a function of load/ unload cycles for the rough dimple and the laser polished dimple. We observe that the plasticity index decreases with an increase in the number of load/unload cycles for the non-polished dimple. In particular, the plasticity index changes from 8.3 to 5.7. However, the plasticity index changes very little with the number of load/unload cycles for the laser polished dimple. This is because the plasticity index was derived from the surface roughness values presented in Fig. 6. This behavior is important for the formation of wear particles and wear.

Figures 8a, b show SEM (Scanning Electron Microscope) pictures of the contact surface of a non-polished dimple and a laser polished dimple after 200 repeated load/ unload cycles, respectively. As can be seen from Fig. 8a, the asperities in the contact region have been flattened. These flatten is due to plastic deformation and is likely to generate wear particles at the dimple/gimbal interface.

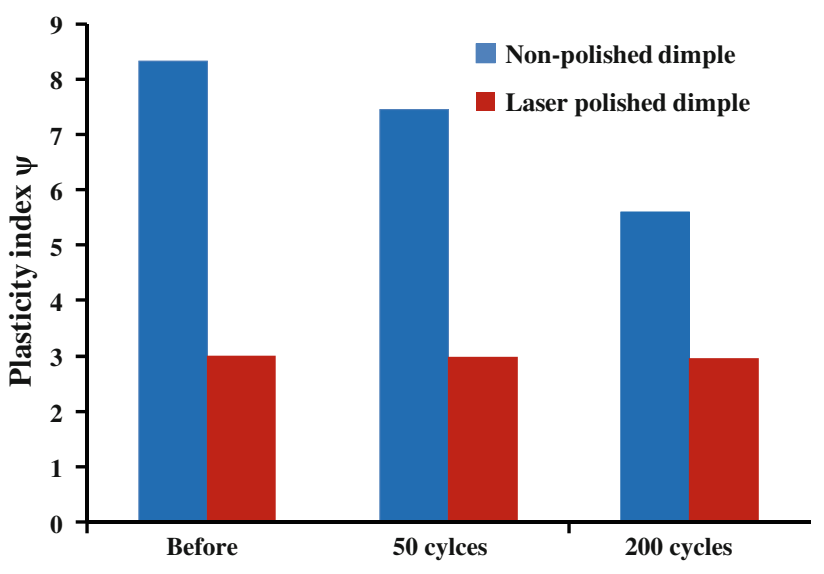

Fig. 7 The plasticity index value as a function of load/unload cycles for a non-polished dimple and a laser polished dimple

Figure 8b shows an SEM image of a laser polished dimple after $200 \mathrm{load} / \mathrm{unload}$ cycles. As can be seen from the figure, very few particles are observed at the dimple surface.

Figure 9 shows the load-displacement characteristics as a function of load cycles for a non-polished dimple and a laser polished dimple contacting a non-polished gimbal, respectively. We observe that the results are similar to the previous results. In particular, we observe that hysteresis is present at the non-polished dimple/non-polished gimbal interface and the laser polished dimple/non-polished gimbal interface and that this hysteresis disappears after a number of load/unload cycles. Very little additional plastic deformation is observed in the elastic shakedown.

Figure 10 shows the load-displacement as a function of load cycles for a non-polished dimple and a laser polished dimple contacting a gold coated gimbal, respectively. We observe again that hysteresis is present. The residual displacement in the first cycle of a dimple contacting a gold coated gimbal is larger than that of a sapphire gimbal shown in Fig. 4 and a non-polished gimbal shown in Fig. 9. This is due to the fact that the gold coated gimbal has lower yield strength than the sapphire gimbal or the non-polished gimbal.

\section{Conclusion}

This paper investigates the contact of the dimple/gimbal interface in a hard disk drive. Based on our experimental results, we observe that:

1. Both dimple types exhibit substantial hysteresis during the first few load/unload cycles. Plastic deformation and hysteresis disappears with subsequent load/unload cycles (elastic shakedown). The plastically deformed asperities may cause wear of dimple/gimbal interface. 
Fig. 8 SEM images for surface of a non-polished dimple a and a laser polished dimple $\mathbf{b}$ after load/unload test
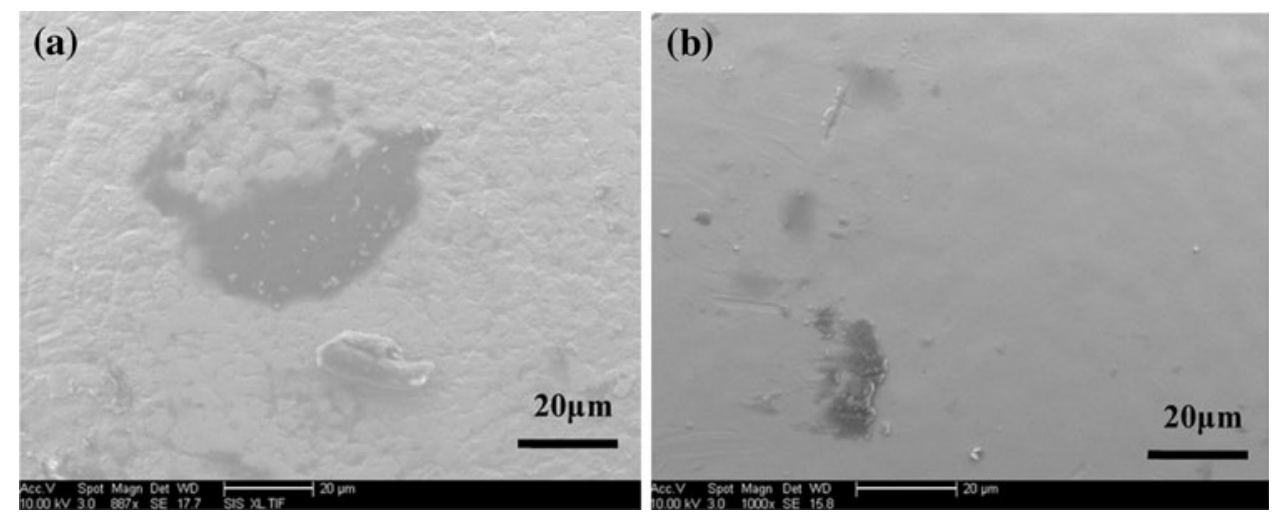
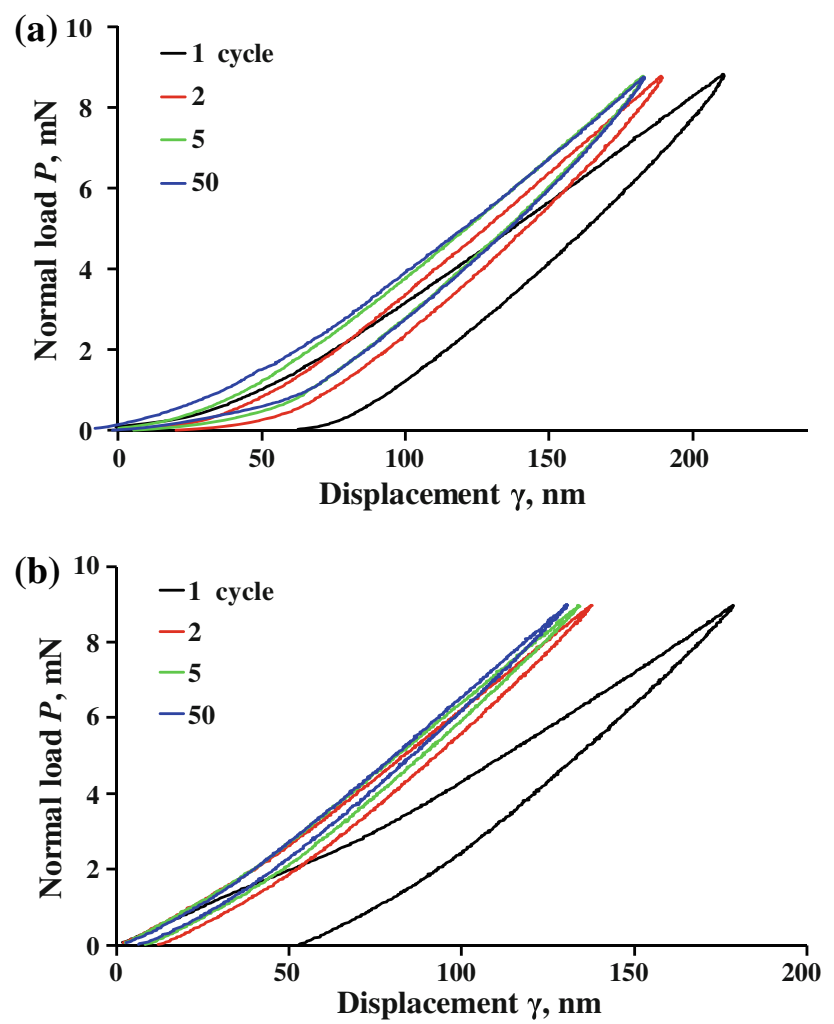

Fig. 9 Normal load versus displacement for different cycles in: a a non-polished dimple and $\mathbf{b}$ a laser polished dimple contacting a nonpolished gimbal, respectively

2. A gold coated gimbal interface shows large plastic deformation in the first few load/unload cycle.

3. The surface roughness and plasticity index of a nonpolished "rough" dimple decrease with an increase in the number of load unload cycles.

4. The surface roughness and plasticity index of a laser polished dimple changes little with the number of load/ unload cycles.

Acknowledgments We would like to thank Mr. Hanya-san of NHK International Corp. for his interest. We also would like to thank Prof. Etsion for his helpful comments and discussions throughout this
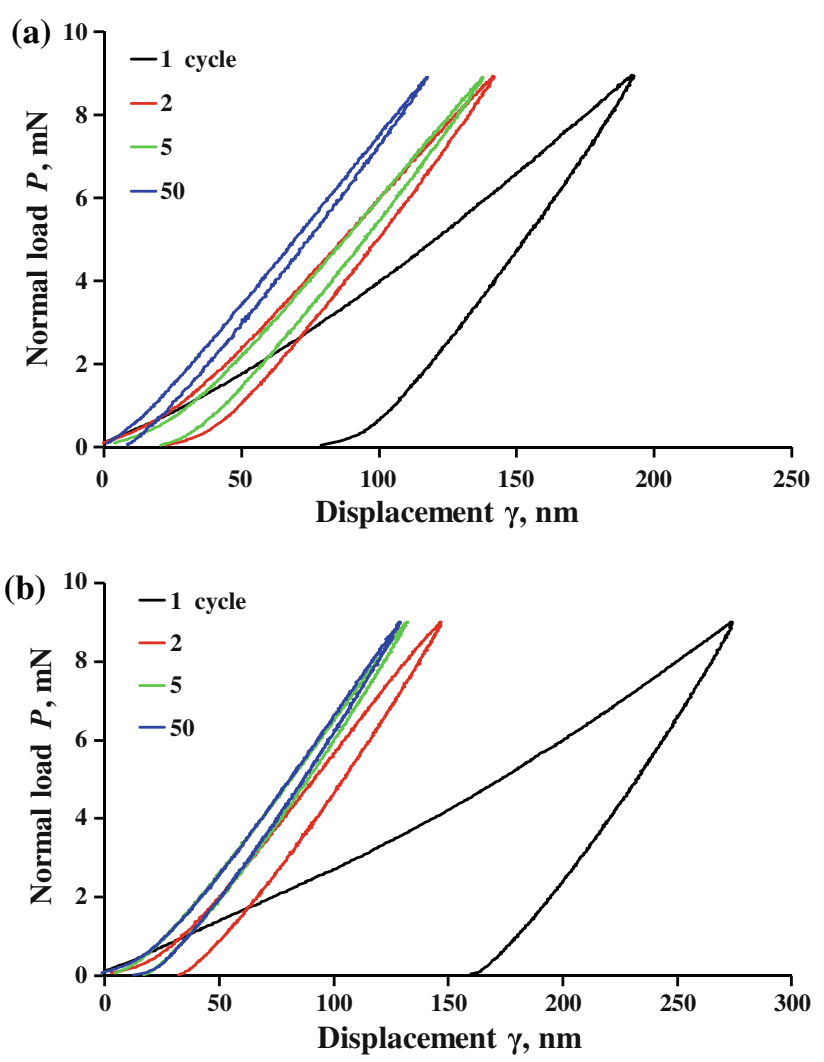

Fig. 10 Normal load versus displacement for different cycles in: a a non-polished dimple and $\mathbf{b}$ a laser polished dimple contacting a gold coated gimbal, respectively

project. L. Li thanks the China Scholarship Council (CSC) and Prof. G. Zhang from Harbin Institute of Technology, for supporting his Ph.D. studies at UCSD.

Open Access This article is distributed under the terms of the Creative Commons Attribution Noncommercial License which permits any noncommercial use, distribution, and reproduction in any medium, provided the original author(s) and source are credited.

\section{References}

Kogut L, Etsion I (2003) A finite element based elastic-plastic model for the contact of rough surfaces. Tribol Trans 46(3):383-390 
König JA (1987) Shakedown of elastic-plastic structures. Elsevier, Amsterdam

Lee DY, Hwang J, Bae GN (2004) Effect of disk rotational speed on contamination particles generated in a hard disk drive. Microsyst Technol 10:103-108

Lee Y, Kim S, Kim K, Park N, Park Y, Kim C, Park K (2009) Analysis of interaction between dimple and flexure by ramp Contact. The magnetic recording conference, Tuscaloosa, pp 81-82

Li L, Etsion I, Fanslau EB, Talke FE (2009) An analysis of the dimple/gimbal contact in a hard disk drive suspension. Proc. of IIP/ISPS Joint MIPE 2009, Yokohama, Japan, pp 105-106
Li L, Ovcharenko A, Etsion I, Talke FE (2010a) Nano-scale displacement of a rough spherical shell loaded against a rigid flat. Proc. of STLE annual meeting \& exhibition, Las Vegas

Li L, Etsion I, Talke FE (2010b) Contact area and static friction of rough surfaces with high plasticity index. Trans ASME J Tribol 132:031401

Raeymaekers B, Helm S, Brunner R, Fanslau EB, Talke FE (2010) Investigation of fretting wear at the dimple/gimbal interface in a hard disk drive suspension. Wear 268:1347-1353

Zheng H, Murthy A, Fanslau EB, Talke FE (2010) Effect of suspension design on the non-operational shock response in a load/unload hard disk drive. Microsyst Technol 16:267-271 